east traditional medicine. It is reported to be popular in the diabetes patients with exuberant heat due to yin deficiency. Health beneficial effects of the dried extract of whole fruits of $C$. grandis (L.) Osbeck var. tomentosa hort. containing $19 \%$ naringin were studied in neonatal streptozotocin (nSTZ)-induced NIDDM rats. The effects of citrus extract and the reference compound sitagliptin were followed on the pancreas histology and on the serum lipid, glucose and insulin levels. The nSTZ treatment led to increased glucose and decreased insulin levels in parallel with marked pancreatic damage. These effects were not observed at least in similar manner in the nSTZ rats given also the citrus extract for several weeks. Beneficial effects of the citrus extract were found on the insulin, glucose and lipid levels as well as on improved pancreatic morphology. The promoting effects seemed to be dependent on an optimal dose since in some measurements they were found especially at certain extract amounts. It is suggested that the treatment of nSTZ diabetic rats with the citrus extract is associated with specific metabolic improvements.

\title{
GASTROPROTECTIVE EFFECT OF FENNEL "FOENICULUM VULGARE" A COMMONLY USED SPICE IN ARAB TRADITIONAL MEDICINE
}

\section{(C) Rafatullah Syed ${ }^{1}$, Alqasoumi Saleh ${ }^{1}$, Al-Dosari Mohammed ${ }^{1}$, Al-Said Mansour ${ }^{1}$, Al-Yahya Mohammed ${ }^{1}$, Al-Mofleh Ibrahim ${ }^{2}$}

\begin{abstract}
${ }^{1}$ Medicinal, Aromatic and Poisonous Plants Research Center(MAPPRC), College of Pharmacy, P. O. Box 2457, King Saud University, Riyadh 11451, Saudi Arabia, Email: srafat@ksu.edu.sa

${ }^{2}$ Department of Medicine, Gastroenterology Unit, College of Medicine, King Khalid University Hospital, P. O. Box 2925, Riyadh-11461, Saudi Arabia
\end{abstract}

Spices are a group of esoteric food adjuncts that have been used for thousands of years to enhance the sensory quality of foods and as preservatives. Much health benefit attributes of these common food adjuncts in animal studies include digestive stimulant action, antioxidant, anti-inflammatory and antihepototoxic properties. Among these, the gastronomic and antioxidant properties of a few specific spices have far-reaching neutraceutical value. The present study was carried out to evaluate antiulcerogenic property of Fennel "Foeniculum vulgare" Mill. (Fam.: Umbelliferae) in Wistar albino rats. An aqueous suspension of fennel (a common dosage form used in folk medicine practice). The suspension was used in two doses ( 250 and $500 \mathrm{mg} / \mathrm{kg}$ body weight, orally) in all experiments except in Shay rat model. Gastric acid secretion studies were undertaken using pylorus ligated (Shay) rats. Gastric lesions in the rats were induced by noxious chemicals including ethanol, strong alkalis and indomethacin. The levels of gastric wall mucus (GWM), nonprotein sulfhydryls (NP-SH) and malondialdehyde (MDA) were also measured in the glandular stomach of rats following ethanol administration. The gastric tissue was also examined histologically. In pylorus-ligated Shay rats, the suspension of fennel significantly reduced the basal gastric acid secretion, titratable acid and ruminal ulceration (64\%, $39 \%$ and $100 \%)$, respectively. The suspension significantly $(P<0.001$, $\mathrm{P}<0.01$ and $\mathrm{P}<0.01)$ attenuated gastric ulceration induced by necrotizing agents $(80 \%$ ethanol, $0.2 \mathrm{~mol} / \mathrm{L}$ $\mathrm{NaOH}, 25 \% \mathrm{NaCl}$ ) respectively and indomethacin was found to be $(P<0.01)$. The cytoprotective and antiulcer effect was further confirmed histologically. Furthermore, the suspension significantly replenished the ethanol-induced depleted levels of GWM $(P<0.001)$, $\mathrm{NP}-\mathrm{SH}(\mathrm{P}<0.05)$ and diminished $(\mathrm{P}<0.01)$ (MDA) concentration of the rats' stomach. The data obtained confirmed the use of fennel suspension in various gastric ailments including stomach ulcers. Fennel reported to contains bio-active phytochemical substances, which might increase endogenous prostaglandins and mucus synthesis and exert its gastroprotective activity through its antioxidant, antisecretory and cytoprotective properties in rats. 